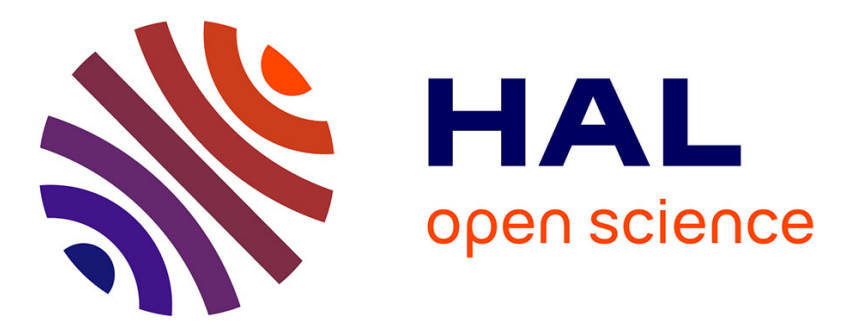

\title{
From physiological principles to computational models of the cortex
}

\author{
Jérémy Fix, Nicolas P. Rougier, Frédéric Alexandre
}

\section{To cite this version:}

Jérémy Fix, Nicolas P. Rougier, Frédéric Alexandre. From physiological principles to computational models of the cortex. Journal of Physiology - Paris, 2007, Neuro-Computation: From Sensorimotor Integration to Computational Frameworks, 101 (1-3), pp.32-39. 10.1016/j.jphysparis.2007.10.009 . inria-00166536v2

\section{HAL Id: inria-00166536 https://hal.inria.fr/inria-00166536v2}

Submitted on 26 Sep 2008

HAL is a multi-disciplinary open access archive for the deposit and dissemination of scientific research documents, whether they are published or not. The documents may come from teaching and research institutions in France or abroad, or from public or private research centers.
L'archive ouverte pluridisciplinaire HAL, est destinée au dépôt et à la diffusion de documents scientifiques de niveau recherche, publiés ou non, émanant des établissements d'enseignement et de recherche français ou étrangers, des laboratoires publics ou privés. 


\title{
From physiological principles to computational models of the cortex
}

\author{
Jeremy Fix* and Nicolas Rougier and Frederic Alexandre \\ Loria, Campus Scientifique, BP 239, 54506 Vandoeuvre-lès-nancy, France
}

\begin{abstract}
Understanding the brain goes through the assimilation of an increasing amount of biological data going from single cell recording to brain imaging studies and behavioral analysis. The description of cognition at these three levels provides us with a grid of analysis that can be exploited for the design of computational models. Beyond data related to specific tasks to be emulated by models, each of these levels also lays emphasis on principles of computation that must be obeyed to really implement biologically inspired computations. Similarly, the advantages of such a joint approach are twofold: computational models are a powerful tool to experiment brain theories and assess them on the implementation of realistic tasks, such as visual search tasks. They are also a way to explore and exploit an original formalism of asynchronous, distributed and adaptive computations with such precious properties as self-organisation, emergence, robustness and more generally abilities to cope with an intelligent interaction with the world. In this article, we first discuss three levels at which a cortical circuit might be observed to provide a modeler with sufficient information to design a computational model and illustrate this principle with an application to the control of visual attention.
\end{abstract}

\section{Motivations}

Building models and frameworks to compute in a biologically inspired way is fruitful for both neuroscience and computer science. On one hand, it leads to simulations that allow a better understanding of the complex relations between structure and function in the brain. Particularly, it is possible to investigate the validity of hypotheses onto these relations. On the other hand, this approach allows to explore a formalism of computation that is hardly used

\footnotetext{
* Corresponding author

Email address: jeremy.fix@loria.fr (Jeremy Fix).
} 
in computer science, based on distributed, asynchronous and adaptive local automata and to learn to master properties such as emergence, unsupervised learning, multimodal processing, robustness, etc. The most critical issue in this process is to get the pertinent information from neuroscience and to select or design the adequate computational principles. The information can be extracted from raw data recorded in nervous systems or in behaving animals. It can also be more elaborated and derive from a more conceptualized source, like a functional model. The computational mechanisms can be derived from a solid mathematical framework (if available) and benefit from its properties (stability, convergence proof). Else, it can be ad hoc mechanisms, suitable for experimental investigations, the theoretical framework of which remains to be built. To implement such a complex task as endowing an autonomous robot with visual search behavior, the interplay between neuroscience and computer science involves several levels of description.

\subsection{The microscopic level}

The microscopic level requires to identify the adequate elementary unit of computation depending on the purpose of the model. For tasks in which the goal is to understand the inner neuronal functioning, either at the level of a single cell or at the level of communication and synchronization between two neurons, spiking neuron models are generally preferred. In tasks like visiomotor coordination involving global patterns of cerebral activity and behavioral assessment, we rely on the mean firing rate of neurons or even on the behavior of elementary circuits of neurons that can be found in structures like the cerebral cortex (Burnod, 1989). Choosing such an intermediate level of description is also fundamental from a computational point of view since handling the temporal behavior of a neuron at the level of the spike is a very consuming task for simulations and is not compatible with the simultaneous evaluation of millions of neurons. Fortunately, mean firing rate models neuronal circuits, as proposed for example by the Continuum Neural Field Theory (Amari, 1977; Taylor, 1999) have proved to be efficient and faithful, compared to cellular recording of population of neurons (like Local Field Potential). Such automata aim at explaining the behavior of the cortical circuitry and generally lay emphasis on the variety of inputs and outputs which are integrated in cortical circuits (Bullier, 2001). Whereas thalamic inputs are generally implemented with a classical integrative model emulating stimulus-specific units (Ballard et al., 1997), cortico-cortical relations are represented as performing a gating effect, implemented with multiplicative connections, and representing feedback as a modulatory activity onto the perceptive flow (Reynolds et al., 2000). Then, the implementation of a cortical area is only specified by the nature of feed-forward and feedback loops feeding a map of interconnected units. The behavior of the whole is only a consequence of patterns of events which 
are presented in the flows and of the interplay of the units. In the simulation, everything is a matter of local numerical computations.

\subsection{The mesoscopic level}

The mesoscopic level is that of cerebral regions, homogeneous at a structural as well as functional level. In the cortex, cortical areas have been detected for a long time, by pure observation of the cytoarchitecture (as soon as the beginning of the 20th century by Brodmann). From that time, a huge quantity of work has been done to relate these areas to a functional role and to gather them in information flows. This has benefited from great progresses in visualization and brain activity measurement techniques (e.g. fMRI, antidromic methods). Sensory and motor poles, and the nature of processing between them have been intensively discussed. Particularly, in the visual case, two main processing flows have been identified from the occipital visual region (Ungerleider and Mishkin, 1982) : one toward the limbic temporal region (ventral pathway) dedicated to visual stimuli identification and the other toward the proprioceptive and parietal regions (dorsal pathway), the role of which is still intensively discussed (Milner and Goodale, 1995), from pure spatial localization to body involvement in visual objects seen as tools. Both temporal and parietal representations are the internal and external sensory representations used by the frontal lobe, seen as the motor pole, responsible for the temporal organization of behavior (Fuster, 1997).

This simplified picture has to be made more complex in several ways. Firstly, instead of sequential processing flows, parallel and redundant processing is reported, in dozens of interconnected cortical areas (Van Essen and Maunsell, 1983; Zeki, 1978) (e.g. color, depth, texture in various areas of the temporal lobe; eye, head and body centered information in the parietal lobe). Secondly, even if this presentation lays emphasis on the feed-forward integration (how to transform visual information into representations of the identity and the location of relevant objects), feedback information seems to play a role at least as important as feed-forward influence (Bullier, 2001) (e.g. receptive fields of neurons in the parietal lobe changing according to body parts orientation (Cohen and Andersen, 2002); the features of a target to look modulate the activity of V4 neurons (Desimone and Duncan, 1995)). Thirdly, our misleading functional and symbolic intuition and the weaknesses of brain imaging techniques incitates us to imagine a step by step processing, where information follows

cycles of processing and builds elaborated representations, whereas the functioning is certainly much more distributed, asynchronous and sparse (Bullier, 2001). 
To better understand and master this counter-intuitive functioning mode, computational models and simulations are of very high interest. From a pure structural description (number and size of areas, connectivity schemes between them) and from necessary functional recommendations (local and asynchronous evaluation of units), the local functioning rules of units (as discussed in the previous section) must be confronted here to the achievement of stable patterns of activity, as observed in the living cortex. This is consequently a way of refinement for the functioning rules of the local automaton. The overall activity pattern which is obtained can also be interpreted as a way to validate the behavioral level, as discussed at the macroscopic level.

\subsection{The macroscopic level}

The macroscopic level is concerned with selecting the task or the behavior you are interested in, and defining the adequate set of areas (together with their connectivity) which is supposed to emulate that task or behavior. Modern imaging techniques and their associated statistical processing offer a valuable tool to relate experimental tasks to brain activations but are not completely satisfactory for several reasons. Firstly, the brain imaging technology itself gives some limitations relative to the kind of behaviors and subjects that can be explored (which are de facto stereotyped), to the parts of the brain easy to observe and to their spatial and temporal resolution. More importantly, observing a pattern of activity in the brain does not give a complete information neither about the role of the recorded region in the behavior nor about the kind of information it stores and processes. More generally, the observed pattern of activity does not provide an interpretation of the underlying cognitive processes. Consequently, these data must be correlated with more behavioral, or even psychological, data and also with brain theories that are themselves elaborated from the synthesis and interpretation of a large quantity of experimental results. In this picture, computational models and simulations are complementary ways of investigation, especially interesting to assess the validity of an hypothesis or to technically explore an intuition. Using the ascendant approach through levels of description, as summarized here, also ensures that the model does not obey a too sequential, centralized, human-like analysis: whatever the possible bias toward such an interpretation, the main constraint is that the simulation has to work in a completely distributed way while yielding an emergent behavior with acceptable spatial and temporal characteristics and with comparable underlying distributed patterns of activity. 

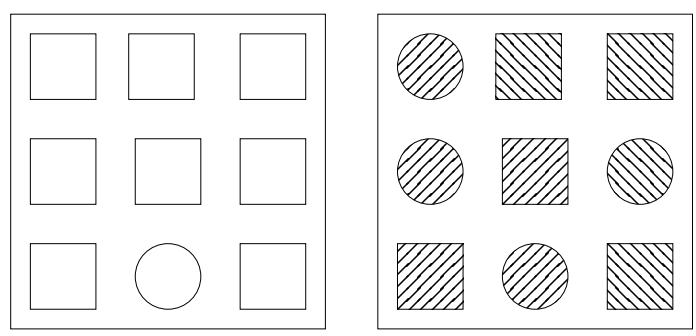

Fig. 1. Feature search can be performed very quickly as illustrated on the left part of the figure; the disc shape literally pops out from the scene. However, as illustrated on the right part of the figure, if the stimuli share at least two features, the pop out effect is suppressed. Hence, finding the disc shape with the stripes going from up-left to down-right requires an active scan of the visual scene.

\section{The computational approach}

The computational approach requires in fact to cope with all these three levels at once in order to have working computational models that can explain or predict some experimental results. However, this is a daunting task since we have to simultaneously integrate data from both anatomy, physiology and psychology. This clearly requires to make clear asumptions and choices at several different levels. We can choose for example among elementary models of the neuron, architectures, granularity of models, adaptive algorithm, etc. As an illustration, we would like to introduce very briefly one widely studied cognitive phenomenom (visual attention) and explain what are the choices we did, what those choices implied on the model and what were their consequences regarding the constraints brought by the framework of distributed, asynchronous, and numerical computations we are using.

\subsection{Psychological and physiological data}

In the early eighties, (Treisman and Gelade, 1980) proposed that the brain actually extracts, in parallel, some basic features from the visual information. Among these basic features, that have been reviewed by (Wolfe, 1998), one can find color, shape, orientation or motion. If we consider a visual search behavior, this task is then equivalent to the finding of conjunction of features that best describes the target. In this sense, (Treisman and Gelade, 1980) distinguished two main paradigms (see also (Duncan and Humphreys, 1989) who proposed a classification of visual search efficiency in terms of targetdistractors similarities):

- Feature search refers to a search where the target sufficiently differs from the distractors to litteraly pop out from the search scene 
- Conjunction search refers to a search where the time to find the target is closely linked to the size of a subset of the search scene, which contains stimuli that are quite similar to the target.

The figure 1 illustrates these two search modes using two tasks whose common goal is to localize a given target. The second task takes more time than the first one and the strategy generally used to perform the task is to successively scan each circle until finding the target. The reaction time then closely depends on the size of the subset of stimuli composed by the circles.

While the pop-out effect can be explained solely on stimulus-driven activities, it must be emphasized that in general, the selection of a subset of potential targets highly depends on the target to look for. This selection process is one component of the more general concept of visual attention. While the brain is submerged by a high quantity of information, and because its ressources are somehow limited, it must perform a selection of the relevant information among what it receives.

In the visual case, this selection mechanism is referred to as visual attention and can take different forms. On the one hand, feature based attention characterises the modulation on the processing of visual information by the knowledge of the features of an object of interest (Motter, 1994). On the other hand, (Rizzolatti et al., 1987) provided evidences for the influence of saccadic eye movements on directed attention, which led to the premotor theory of attention. (Moore and Fallah, 2001) have also shown that the preparation of an eye movement toward a specific location provides a bias to the cells whose receptive field covers that location. This spatial bias is known as spatial attention. Several experiments have provided evidences that our brain can provide such a spatial bias covertly in the absence of the overt deployment of eye movements (Posner et al., 1980), and that the underlying circuits mediating the covert and overt deployment of attention might considerably overlap (Awh et al., 2006).

The first neural correlate of visual attention at the single cell level has been discovered by Moran et al.(Moran and Desimone, 1985) in V4 where neurons were found to respond preferentially for a given feature in their receptive field. When a preferred and a non-preferred stimulus for a neuron are presented at the same time in its receptive field, the response becomes an average between the strong response to the preferred feature and the weak response to the nonpreferred one. But when one of the two stimuli is attended, the response of the neuron represents the attended stimulus alone (strong or poor), as if the nonattended were ignored. Attentional modulation of neuronal activity was also observed in other cortical areas. In (Treue and Maunsell, 1996), the author reported feature-based attentional modulation of visual motion processing in 

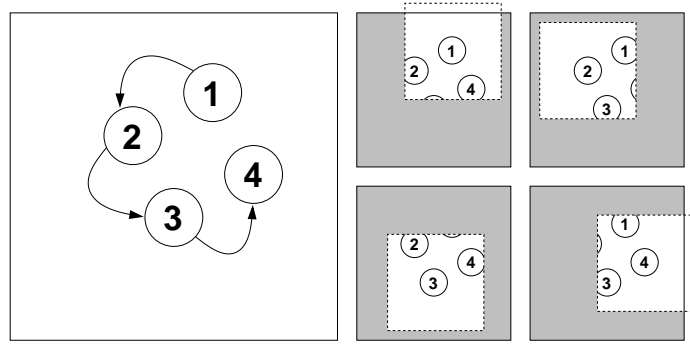

Fig. 2. When scanning a visual scene, going for example from stimulus 1 to stimulus 4 , as illustrated on the left of the figure, the image received on the retina is radically changed after each eye movement. When the task requires to memorize the positions of the previously focused stimuli, the difficulty is to be able to update their memorised positions after each saccade. The figures on the stimuli are shown only for explanation purpose and do not appear on the screen; all the stimuli are identical.

area MT. An increasing litterature is also reporting that the modulatory effect of attention is not restricted to the extrastriate cortex but also extends to the early visual areas (Silver et al., 2007).

The observed modulatory effect of attention on the processing of single units raises the intriguing issue of determining its origin(s). As detailed in the introduction, the processing of visual information is supposed to rely on two pathways. On the one hand, the ventral pathway, going from the occipital lobe through the temporal lobe is classicaly thought to mediate object recognition (Gross, 1994). Several studies have shown the influence of the intrinsic properties of an object of interest on the processing of single cells (Chelazzi et al., 1998). This feature-based mechanism could originate from the ventral pathway via massive feedback connections (Rockland and van Hoesen, 1994), and might be generated in the ventrolateral prefrontal cortex to provide a bias corresponding to the features of an object of interest. On the other hand, the dorsal pathway going from the occipital lobe through the parietal lobe is supposed to be involved in producing motor representations of sensory information for the purpose of guiding movements (Cohen and Andersen, 2002; Matelli and Luppino, 2001). The temporal properties of neurons in the parietal cortex cannot be solely explained by proprioceptive feedbacks as a consequence of a performed movement. Rather, anterior areas might provide more posterior areas with the parameters of an impeding movement, then leading to anticipatory activations or remapping, as observed by Colby et al.(Merriam and Colby, 2005; Merriam et al., 2007). The later have shown that, in the case of saccadic eye movements, neurons in lateral intraparietal area (LIP) exhibit saccade-related activity occuring before, during and/or after a saccade bringing a stimulus in the receptive field of the recorded neurons. These recordings reveal that a circuit, involving parietal areas, is able to predict the future position of currently observed stimuli after an impeding eye movement. 
Moreover, in the case of overt deployment of attention, a crucial issue is to be able to update the position of previously attended stimuli after each saccade (see figure 2).

Saccadic eye movements are too fast to suppose that a memory of the targets can be continuously updated with the visual input. Hence, a specific mechanism using the memorized locations of the targets and an impeding eye movement, predicting the future positions of these targets must exist. The frontal eye field (FEF) might be involved in such a circuit. As shown by (Sommer and Wurtz, 2004), FEF receives projections from the superior colliculus (SC), relayed by the mediodorsal thalamus, which could convey a corollary discharge of movement commands. Several studies have also shown memory related activity in FEF (Lawrence et al., 2005) as well as predictive responses (Umeno and Goldberg, 1997). This illustrates that the brain consists in several cooperating areas and that a behavior observed in tasks such as a visual search actually emerges from distributed computations.

\subsection{Computational approaches to visual attention}

In the field of computational neuroscience, several attempts at modeling visual attention have been proposed. The pioneering work of (Koch and Ullman, 1985), relying on the Feature Integration Theory(Treisman and Gelade, 1980), distinguishes several channels extracted from the visual input (color, orientation, intensity), each of them represented in different sets of maps, used to build conspicuity maps to finally lead to a single spatial map representing the behavioral relevance of each location in the visual field, the so-called saliency map. The selection of a location to attend to is then determined by a winnertake-all operation on the saliency map. A memory of the attended locations finally biases that winner-take-all computation to avoid attending to previously attended locations. This phenomenon reflects one component of the inhibition-of-return introduced by Posner and detailed in the previous section : a cued location facilitates the deployment of attention at that location when the time between the cue and the target is short, but, for longer delays, we observe the reverse effect and, if the target is presented at a cued location, its processing takes longer. The model proposed by Koch and Ullman was the first step to further developments (Itti and Koch, 2001) but, from the past few years, we are observing a slight shift from purely feedforward models to models using both feedforward and feedback projections (Tsotsos et al., 1995), since it is now widely accepted that feedback influences play a crucial role in single unit processing. Among these models, we will focus in the rest of this article on the work of (Hamker, 2004). This model clearly distinguishes between the ventral and dorsal pathways with a feature-based and a spatial 
stream processed along two separate pathways. It also emphasizes the role of feedback projections that are supposed to be the cause of attentional effects. The ventral stream provides a feature-based bias corresponding to an object of interest (an object we are looking for in a visual search task for example) and the dorsal stream provides a spatial bias corresponding to a region of interest, that might be the target for an impeding eye movement. The main hypothese is that V4 could be an intermediate layer, being the major source of information carrying along the ventral and dorsal pathways, and the major target of projections from higher cortical areas. The proposed model exhibits good performances in visual search task but one of the limitations is that the model is restricted to the covert deployment of attention, where no eye movement is initiated. We will see in the following sections a possible extension of this approach to deal with saccadic eye movements.

\subsection{A computational model}

The models we propose are built in the framework of local, distributed, asynchronous and numerical computations by considering assemblies of units that we call maps, each unit being connected with other units in the same map by lateral connections and with units from other maps by afferent connections. A unit is a stand-alone computational element, characterised by a numeric activity $u_{M}(\mathbf{x}, t)$ that is locally updated by computing the influence of input units. The activity of each unit follows the ordinary differential equation (1) coming from the Continuum Neural Field Theory (Amari, 1977).

$$
\begin{aligned}
u_{M}(x, t+1) & =u_{M}(x, t)+\tau \cdot \delta u_{M}(x, t) \\
\delta u_{M}(x, t) & =\sum_{y \in M} w_{x y} \cdot u_{M}(y, t)+I(x)
\end{aligned}
$$

where $M$ and $M^{\prime}$ are maps of units and $I(x)$ is a function computing the influence of afferent units.

A key point is to determine how the cells combine their inputs to perform their local computations. V4 neurons are a striking example of attentional modulation at the single cell (or small population) level, as explained in the previous section. Let us consider a population of orientation selective cells, receiving afferent connections from lower level areas, these connections being directly modulated by feedback connections coming from higher level areas. These feedback projections carry information about the features of an object of interest (feature based attention) and a location that might be the target of an impeding action (spatial attention) that have been shown to have an influence on the response of V4 neurons. In (Taylor et al., 2006; Reynolds et al., 2000), the authors show that, among different possibilities of integration 


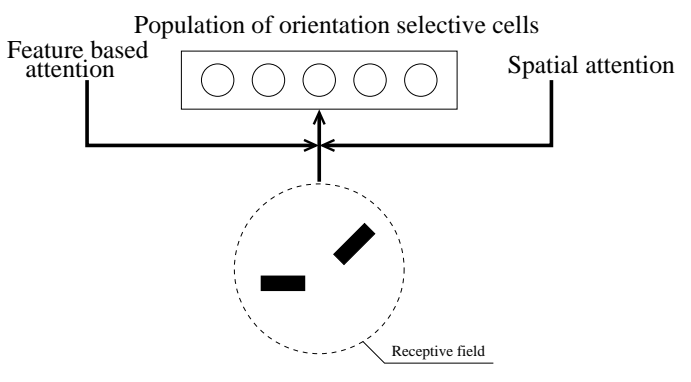

a)

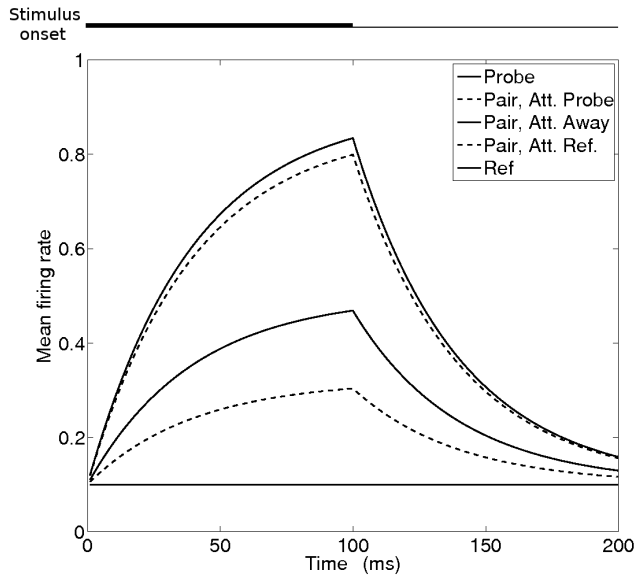

b)

Fig. 3. a) A population of orientation selective cells sharing the same receptive field. The afferent connections are modulated by feature-based and spatial attention as proposed in the contrast gain model b) When a prefered and non-prefered stimuli are both presented in the receptive field, the response of the neuron is an average between the responses to the stimuli presented separately. When feature-based attention is directed either toward one or the other stimulus, the cells respond as if only the attended stimulus was present. This effect is even stronger when spatial attention is directed toward the receptive field. The plots are displayed in the same order than the legend.

of the feedback modulation, the contrast gain model seems to be the most suitable (figure 3a). In this model, if we record the activity of one unit while presenting two stimuli in the receptive field of the population (a preferred and a non-preferred stimulus for the considered unit), we observe two properties (figure $3 b)$ :

- attending the preferred stimulus drives the activity of the cell toward its response when only the preferred stimulus is presented

- attending the non-preferred stimulus drives the activity of the cell toward its response when only the non-preferred stimulus is presented

These modulatory effects reflect the biased competition mechanism introduced by (Desimone and Duncan, 1995) and illustrate how we can deal with biological data at the single-cell level.

Let us now consider modeling at a higher level, gathering elementary computational units to form maps. These maps combine flows of information and cooperate in a distributed way to allow the emergence of a global behavior. As an illustrating example, let us consider the mechanisms with which the brain might memorize the attended locations and update these positions after each eye movement, in the case of an overt deployment of attention (figure 


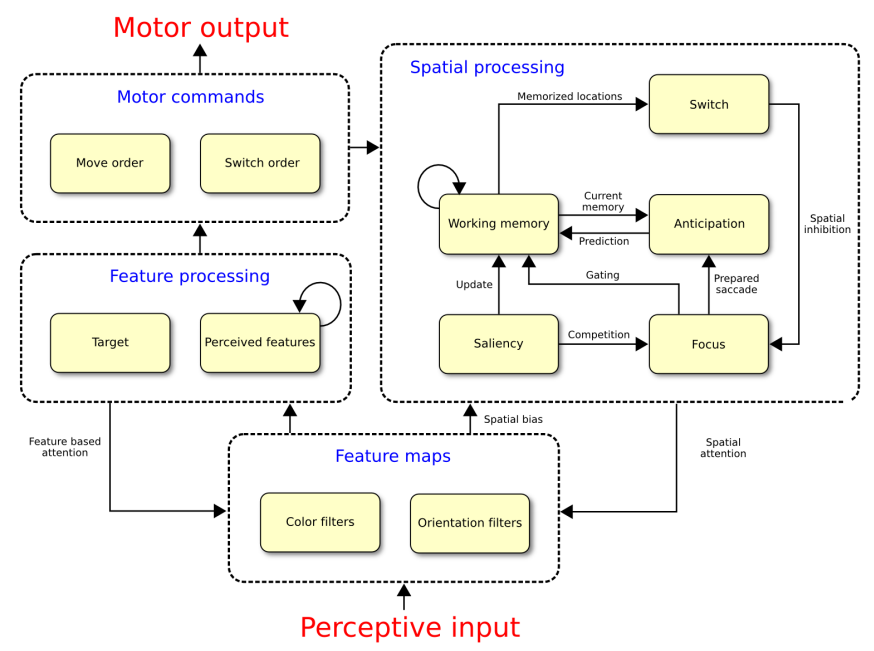

Fig. 4. An example of model relying on local, distributed, asynchronous and numerical computations, used to perform a visual search task. Further details can be found in the text below.

2). In (Vitay and Rougier, 2005), we have proposed to connect homogeneous assemblies of units to build a dynamic working memory circuit. We have extended this model in (Fix et al., 2006) to take into account the eye movements while performing a visual search task, by adding a mechanism that predicts the consequences of these saccades on the visual perception. We have shown that disrupting this mechanism drastically impairs the performances of the system. At the single cell level, these models are homogeneous and are built with the same basic units. The specificity of each map only comes from the pattern of connections that connect it to the other maps. The structure of these projections defines the architecture at a mesoscopic level.

We can also think about a model as a whole, and use it to perform visual search tasks, measuring psychological variables as, for example, the reaction time. Let us consider the model depicted on figure 4. This figure illustrates how the models proposed in (Hamker, 2004) and (Fix et al., 2006) could be combined, leading to one among other possibilities of computational models that gather the psychological and physiological data detailed in the previous sections. The purpose of this article is not to explain deeply the patterns of connections between the maps. Rather, we would like to emphasize how the flows of information are combined to allow the emergence of a behavior in a distributed architecture. The interested reader can find a complete description of the models in (Hamker, 2004) and (Fix et al., 2006).

The visual input is processed in parallel in different maps, extracting basic features. This distributed representation of the visual input, labeled Feature Maps, then feeds two pathways, a spatial non-feature specific one and a feature roughly non-spatial one. The main purpose of the first is to spatially select a location of interest (within the Saliency and Focus maps), to memorize that that given location has been attended to (the memory consists in 


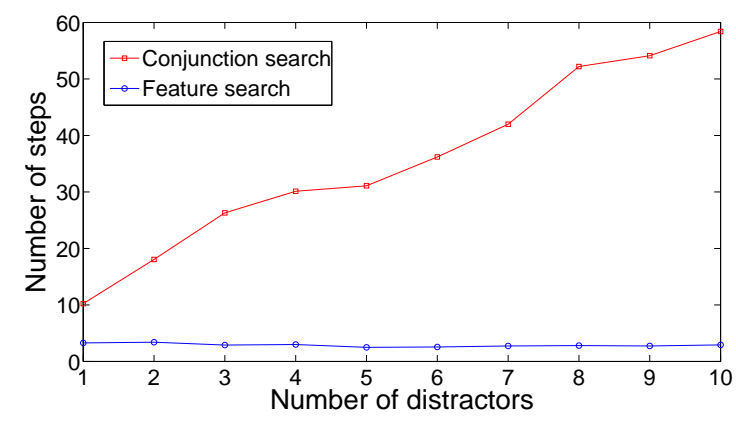

Fig. 5. The reaction time, defined as the number of computational steps requiered to perform the task, increases linearly with the set size in the conjunction search paradigm while keeping constant in the feature search paradigm.

a recurently connected circuit labeled working memory), and to anticipate the consequences of an eye movement on this memory, if the movement is triggered (with the Anticipation map). A key point of the model is the use of feedback projections of the selected location to the Feature maps, biaising this distributed representation toward the features of the stimulus at the attended location. The feature specific pathway then combines this representation with a target template. This template might be a complex combination of basic features and is also projected via feedback connections to the Feature maps. The resulting activities in the Feature processing maps is then propagated to the decision area so as to provide it with the necessary clues to decide which behavior to adopt. In our case, we distinguish two decisions : one is to switch covertly the locus of attention (covert attention) and the other is to perform an eye movement toward that location (overt attention). When an eye movement is performed, the target is decoded from the Focus map. A striking consequence of the distributed nature of the computations is that the memory is fed with an attended location at the same time that the decision to switch covertly or overtly the attention is taken.

If we now use this model to perform a visual search task ${ }^{1}$ and see it as a black box, we can restrict the measurements to the available ones from the point of view of an external observer, as it would be done by psychologists performing this kind of task with monkeys. We can for example measure the time it takes for the model to perform the task. In a task involving eye movements, we can also record the number of saccades performed by the "subject", the target of the movements, the scanpath, etc.. The figure 5 represents the reaction time, function of the set size, in the two paradigms of feature search and conjunction search. In the first case, the task is to detect a blue bar, among green bars. In the second case, the task is to detect a blue bar at 45 degrees among distractors that share at least one feature with the target, namely green bars

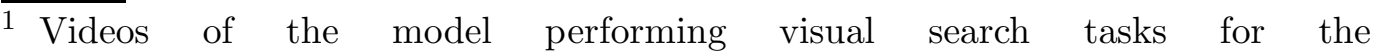
two paradigms of feature and conjunction search are available at http://www.loria.fr/ rougier/index.php?n=Demos.Demos
} 


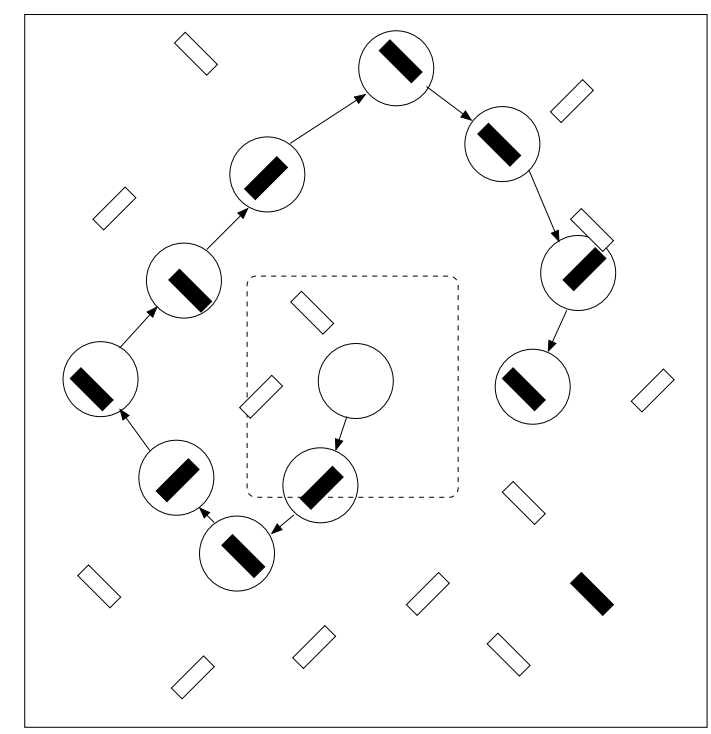

Fig. 6. Example of scanpath obtained during a search in which the model has to perform an eye movement toward each black target. The dashed rectangle represents the visual field and the circles represent its successive positions. The target at the bottom right is never focused since it never appears in the visual field.

at 45 degrees or blue bars at 135 degrees. We can then observe a classical set size effect : in a feature search, the time to perform the task does not depend on the number of distractors whereas the time to perform a conjunction search linearly depends on the set size.

The figure 6 is an illustration of a scanpath obtained during a visual search task in which the model has to perform an eye movement toward each of the black targets, the visual field being limited to the dashed rectangle ${ }^{2}$. The working memory contains all the previously focused stimuli and is updated after each movement. It thus provides the selection process with an inhibitory bias so that, when several targets appear in the visual field, the next selected target is necessarily a non-previously focused one.

\section{Discussion}

The interplay between neuroscience and computer science clearly needs to be reinforced if we want to go any further in our understanding of cognition. This is one of the goals of the field of computational neurosciences that aims ultimately at gathering knowledge and expertise from several domains to propose

$\overline{2}$ A video of the model performing a visual search task with explicit eye movements is available at http://www.loria.fr/ rougier/index.php?n=Demos.Demos 
new theories for brain and cognition. This article highlights a possible way of bridging the gap between computer science and neuroscience by explaining what are the interests and the constraints of modeling and how to cope with the huge amount of available data from psychological experiment, fMRI, single cell recording, etc. We have to make hypothesis and choices without necessarily having the legitimacy to do so. However, we think that having such a strongly constrained and clearly defined modeling framework helps us to make the right asumptions. In this sense, we clearly try to restrict ourselves to the design of the most simple model that can explain data without strong considerations for an exact model. For example, we know that communication between neurons is done using spike trains while we are using mean-firing rate models of neuron. At the single cell level, this would be a hardly-defendable position since we cannot take into account a wide range of phenomena that are known to happen at this scale. However, at the functional level, where virtually thousands of such units are interacting together, this assumption makes sense and helps us to have a better understanding of the whole. Of course, a question remains on how properties of a functional model would cope with a more detailed model of neurons. Would it change fundamentally or would it be rather a refinement of the existing properties: the strength of computational models is to have the opportunity to refine this level of description, to compare it with more precise observations, without drawing again the whole system.

At the mesoscopic level, modeling meets neuroscience on the analysis of implicated populations and of their underlying behavior. Similarly to the refinement process in neuroscience that corresponds to iteratively better understand the functional role of a cortical map in a task, computational models can also enrich their description of maps of computing units, seen as the crossroads of feed-forward, feed-back and lateral information flows. At this level, adding learning rules, designed as the way to describe the mutual influence of these flows, is certainly the most important task to consider in the near future.

The behavior of computational models at the macroscopic level is intended to have a deep impact in the behavioral neuroscience and to offer them a new behaving entity on which to apply their measurement and analysis. This can be particularly interesting if the simulations are embedded in such autonomous agents as robots, giving a direct access to an embodied cognition.

\section{References}

Amari, S., 1977. Dynamics of pattern formation in lateral-inhibition type neural fields. Biological Cybernetics 27, 77-87.

Awh, E., Armstrong, K., Moore, T., 2006. Visual and oculomotor selection: 
links, causes and implications for spatial attention. Trends in Cognitive Sciences 10 (3), 124-130.

Ballard, D., Hayhoe, M., Pook, P., Rao, R., 1997. Deictic codes for the embodiment of cognition. Behavioral and Brain Sciences 20, 723-742.

Bullier, J., 2001. Integrated model of visual processing. Brain Research Reviews 36 (2-3), 96-107.

Burnod, Y., 1989. An adaptive neural network : the cerebral cortex.

Chelazzi, L., Duncan, J., Miller, E., Desimone, R., 1998. Responses of neurons in inferior temporal cortex during memory-guided visual search. Journal of Neurophysiology 80 (6), 2918-2940.

Cohen, Y., Andersen, R., 2002. A common reference frame for movement plans in the posterior parietal cortex. Nature Review Neuroscience 3 (7), 553-62.

Desimone, R., Duncan, J., 1995. Neural mechanisms of selective visual attention. Annual Review of Neuroscience 18, 193-222.

Duncan, J., Humphreys, G., 1989. Visual search and stimulus similarity. Psychological Review 96 (3), 433-58.

Fix, J., Vitay, J., Rougier, N., 2006. Anticipatory Behavior in Adaptive Learning Systems : From Brains to Individual and Social Behavior. Springer Verlag, LNAI 4520, Ch. A distributed computational model of spatial memory anticipation during a visual search task.

Fuster, J., 1997. The Prefrontal Cortex : Anatomy, Physiology, and neuropsychology of the frontal lobe.

Gross, C., 1994. How inferior temporal cortex became a visual area. Cerebral Cortex 4, 455-469.

Hamker, F., 2004. A dynamic model of how feature cues guide spatial attention. Vision Research 44, 501-521.

Itti, L., Koch, C., 2001. Computational modeling of visual attention. Nature Reviews Neuroscience 2 (3), 194-203.

Koch, C., Ullman, S., 1985. Shifts in selective visual attention : Towards the underlying neural circuitry. Human Neurobiology 4 (4), 219-227.

Lawrence, B., White, R., Snyder, L., 2005. Delay-period activity in visual, visuomovement, and movement neurons in the frontal eye field. Journal of Neurophysiology 94, 1498-1508.

Matelli, M., Luppino, G., 2001. Parietofrontal circuits for action and space perception in the macaque monkey. NeuroImage 14, S27-S32.

Merriam, E., Colby, C., 2005. Active vision in parietal and extrastriate cortex. The Neuroscientist 11 (5), 484-493.

Merriam, E., Genovese, C., Colby, C., 2007. Remapping in human visual cortex. Journal of Neurophysiology 97, 1738-1755.

Milner, D., Goodale, M., 1995. The visual brain in action.

Moore, T., Fallah, M., 2001. Control of eye movements and spatial attention. PNAS 98 (3), 1273-1276.

Moran, J., Desimone, R., 1985. Selective attention gates visual processing in the extrastriate cortex. Science 229, 782-784.

Motter, B., 1994. Neural correlates of attentive selection for color or luminance 
in extrastriate area v4. Journal of Neuroscience (14), 2178-2189.

Posner, M., Snyder, C., Davidson, B., 1980. Attention and the detection of signals. Journal of Experimental Psychology 109 (2), 160-74.

Reynolds, J., Pasternak, T., Desimone, R., 2000. Attention increases sensitivity of v4 neurons. Neuron 26, 703-714.

Rizzolatti, G., Riggio, L., Dascola, I., Ulmita, C., 1987. Reorienting attention accross the horizontal and vertical meridians. Neuropsychologia 25, 31-40.

Rockland, K., van Hoesen, G., 1994. Direct temporal-occipital feedback connections to striate cortex (v1) in the macaque monkey. Cerebral Cortex 4, 300-313.

Silver, M., Ress, D., Heeger, J., 2007. Neural correlates of sustained spatial attention in human early visual cortex. Journal of Neurophysiology 97, 229237.

Sommer, M., Wurtz, R., 2004. What the brain stem tells the frontal cortex. i. oculomotor signals sent from superior colliculus to frontal eye field via mediodorsal thalamus. Journal of Neurophysiology 91, 1381-1402.

Taylor, J., 1999. Neural bubble dynamics in two dimensions. Biological Cybernetics 80, 5167-5174.

Taylor, N., Harthley, M., Taylor, J., 2006. The micro-structure of attention. Neural Networks 19, 1347-1370.

Treisman, A., Gelade, G., 1980. A feature-integration theory of attention. Cognitive Psychology 12 (1), 97-136.

Treue, S., Maunsell, J., 1996. Attentional modulation of visual motion processing in cortical areas mt and mst. Nature 382, 539-541.

Tsotsos, J., Culhane, S., Lai, W., Davis, N., 1995. Modeling visual attention via selective tuning. Artificial Intelligence 78, 507-545.

Umeno, M., Goldberg, M., 1997. Spatial processing in the monkey frontal eye field. i. predictive visual responses. The American Psychological Society 78, $1373-1383$.

Ungerleider, L., Mishkin, M., 1982. Analysis of Visual Behavior. MIT Press, Ch. Two cortical visual systems, pp. 549-586.

Van Essen, D., Maunsell, J., 1983. Hierarchical organization and functional streams in the visual cortex. Trends Neuroscience 6, 370-375.

Vitay, J., Rougier, N., 2005. Using neural dynamics to switch attention. In: International Joint Conference on Neural Networks. IJCNN.

Wolfe, J., 1998. Visual search. In: Attention. University College London Press.

Zeki, S., 1978. Functional specialisation in the visual cortex of the rhesus monkey. Nature 274, 423-428. 